\title{
OPEN Using noise to augment synchronization among oscillators
}

\author{
Jaykumar Vaidya, Mohammad Khairul Bashar \& Nikhil Shukla
}

Noise is expected to play an important role in the dynamics of analog systems such as coupled oscillators which have recently been explored as a hardware platform for application in computing. In this work, we experimentally investigate the effect of noise on the synchronization of relaxation oscillators and their computational properties. Specifically, in contrast to its typically expected adverse effect, we first demonstrate that a common white noise input induces frequency locking among uncoupled oscillators. Experiments show that the minimum noise voltage required to induce frequency locking increases linearly with the amplitude of the oscillator output whereas it decreases with increasing number of oscillators. Further, our work reveals that in a coupled system of oscillators-relevant to solving computational problems such as graph coloring, the injection of white noise helps reduce the minimum required capacitive coupling strength. With the injection of noise, the coupled system demonstrates frequency locking along with the desired phase-based computational properties at $5 \times$ lower coupling strength than that required when no external noise is introduced. Consequently, this can reduce the footprint of the coupling element and the corresponding areaintensive coupling architecture. Our work shows that noise can be utilized as an effective knob to optimize the implementation of coupled oscillator-based computing platforms.

Coupled oscillators have experienced renewed interest in computation owing to their rich spatial-temporal properties $^{1,2}$. Besides their potential application in realizing associative memory ${ }^{3,4}$ and oscillator neural networks $(\mathrm{ONNs})^{5-8}$ for tasks such as image processing ${ }^{9,10}$, these systems have recently been explored for solving hard combinatorial optimization problems which are still considered intractable to solve using conventional digital computers. Examples of such problems include graph coloring ${ }^{11}$ (representative problem considered here), computing the maximum independent set ${ }^{12}$ and maximum cut of a graph ${ }^{13-16}$ among others. While coupled oscillators can provide an alternate, and potentially more efficient, pathway to solving such problems, one of the important factors in the design and implementation of such analog systems is noise. Normally, the injection of external noise should have adverse effects on the performance of electronic circuits with analog circuits such as oscillators being particularly susceptible. In fact, this was an important consideration in the adoption of digital circuits over analog ones in the $1950 \mathrm{~s}^{17}$.

However, in contrast to its typically undesirable effects, noise can play a constructive role in promoting the highly non-linear process of synchronization among oscillators. Prior work has studied the effects of different types of noise on the synchronization of oscillators, both, theoretically (example ${ }^{18-27}$, ) and experimentally (example ${ }^{28-30}$,); identical and non-identical oscillators have been shown to exhibit synchronization in response to both white and colored noise. Noise induced synchronization has been explored in various neural networks ${ }^{31-33}$. In fact, the effect of noise on synchronization has even been explored in biological systems such as spike generation in neurons of neocortical slices of rats $\mathrm{s}^{34}$, firing patterns of two uncoupled neurons in paddlefish ${ }^{35}$, as well as in other physical systems such as lasers ${ }^{36,37}$. However, the effect of noise injection on the synchronization of oscillators in the context of their computational properties, particularly for solving combinatorial optimization problems (here, graph coloring), remains largely unexplored. Therefore, in this work, we investigate using experiments and simulations, the role of noise in the coupling dynamics of oscillators and on their resulting computational properties. Specifically, we demonstrate that the injection of noise lowers the minimum coupling strength required to induce frequency locking and the subsequent phase properties required for computation.

\section{Results}

Synchronization of two uncoupled oscillators. We first investigate the effect of noise on the synchronization of two uncoupled oscillators with a common (white) noise input. Figure la shows the schematic of a Schmitt-trigger based relaxation oscillator along with the component values used in the discrete (breadboardbased) experimental realization; the Schmitt-trigger is designed using an OPAMP (LM741) and the oscillations 


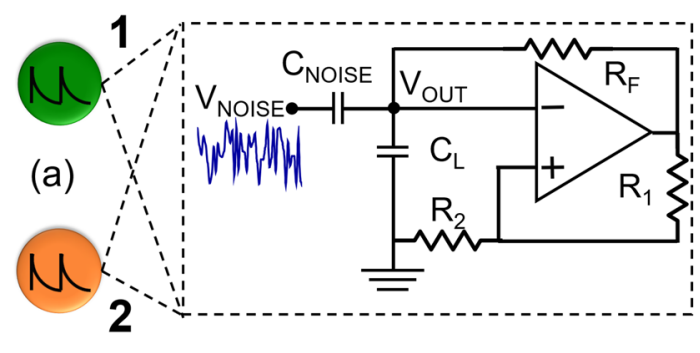

\begin{tabular}{|c|c|}
\hline Parameter & Value \\
\hline$R_{1}$ & $6 \mathrm{k} \Omega$ \\
$\mathrm{R}_{2}$ & $80 \mathrm{k} \Omega$ \\
\hline $\mathrm{R}_{\mathrm{F}}$ & $(330 \pm 1) \mathrm{k} \Omega$ \\
\hline $\mathrm{C}_{\mathrm{L}}$ & $100 \mathrm{pF}$ \\
\hline $\mathrm{C}_{\mathrm{NOISE}}$ & $1 \mathrm{pF}$ \\
$\mathrm{V}_{\mathrm{DD}}$ & $3 \mathrm{~V}$ \\
\hline
\end{tabular}

\section{(b) No injected Noise: No Frequency Synchronization}


\section{(d) $V_{\text {noise }}=270 \mathrm{mV}$ (@ threshold): Frequency Synchronized}

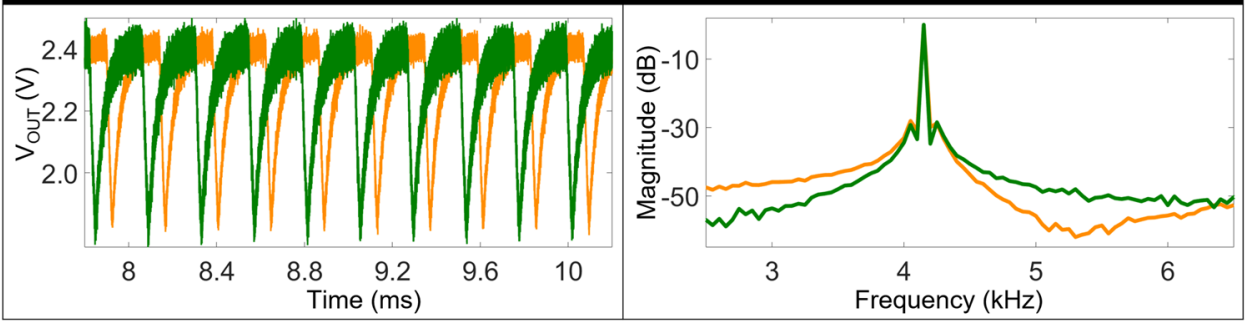

Figure 1. (a) Schematic of the Schmitt-trigger oscillator along with the values of the components used in the experimental realization. Time domain output and frequency spectrum of the two oscillators for various white noise inputs: (b) No noise; (c) $\mathrm{V}_{\mathrm{NOISE}}=100 \mathrm{mV}_{\mathrm{RMS}}$; (d) $\mathrm{V}_{\mathrm{NOISE}}=270 \mathrm{mV}_{\mathrm{RMS}}$. No frequency locking is observed until $\mathrm{V}_{\text {NOISE }} \geq 270 \mathrm{mV}_{\text {RMS }}$.

are stabilized using a negative RC feedback. The frequency of oscillations can be tuned using the resistor $\left(\mathrm{R}_{\mathrm{F}}\right)$ and the capacitor $\mathrm{C}_{\mathrm{L}}$ in the negative feedback loop; we intentionally introduce a small change in $\mathrm{R}_{\mathrm{F}}$ of the two oscillators to ensure they have slightly different frequencies and are not synchronized trivially. White noise generated using a function generator (Keysight $81160 \mathrm{~A}$ ) is injected at the output of the oscillator through a capacitor $\left(\mathrm{C}_{\mathrm{NOISE}}=1 \mathrm{pF}\right)$; the value $\mathrm{C}_{\mathrm{NOISE}}$ is chosen such that the oscillators, in the absence of noise, do not exhibit frequency locking using only $\mathrm{C}_{\mathrm{NOISE}}$. The oscillator outputs are measured using a digital oscilloscope (Keithley DSO104A).

Figure $1 \mathrm{~b}-\mathrm{d}$ shows the time domain waveforms and the corresponding frequency spectrum of the oscillators when subjected to different levels of external white noise. In the absence of externally injected noise (Fig. 1b), no frequency locking is observed among the oscillators. Furthermore, the oscillators fail to frequency lock (Fig. 1c) 

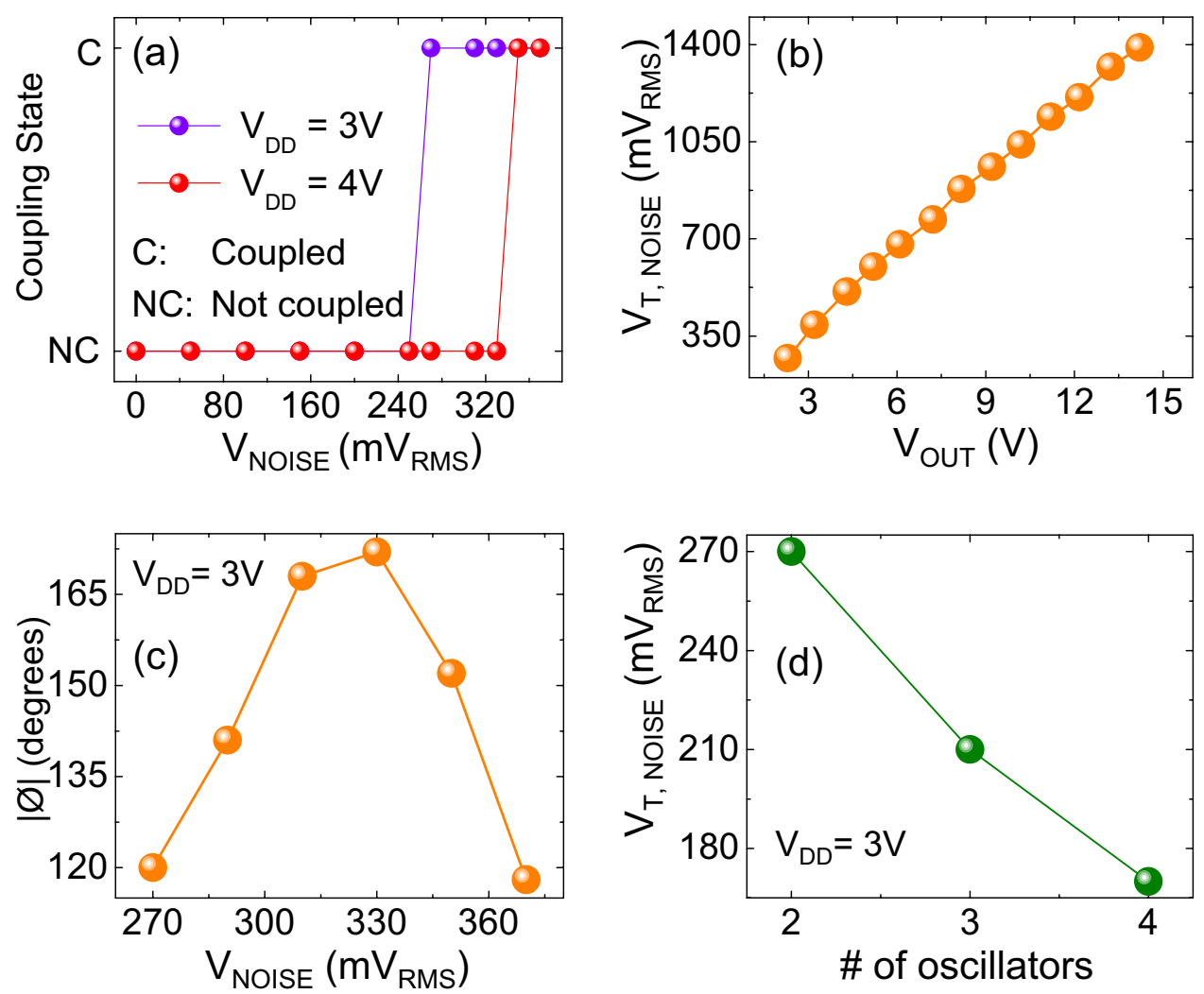

Figure 2. (a) Effect of noise voltage on the synchronization of the two oscillators for two different biases $\left(\mathrm{V}_{\mathrm{DD}}=3 \mathrm{~V}, 4 \mathrm{~V}\right)$. (b) Variation of noise threshold (for frequency locking) with amplitude of the output. (c) Evolution of the relative phase difference $(\varnothing)$ between the synchronized oscillators as function of the noise voltage. (d) Minimum noise threshold $\left(\mathrm{V}_{\mathrm{T}, \mathrm{NOISE}}\right)$ required for synchronization as a function of the number of oscillators; the threshold decreases with increasing number of oscillators.

until the noise voltage reaches the threshold value of $270 \mathrm{mV}_{\mathrm{RMS}}$ (Fig. 1d) even though the frequency mismatch among the oscillators decreases as the intensity of the injected noise is increased. However, when the noise voltage equals or exceeds this threshold input, the oscillators exhibit (noise induced) frequency locking as evident by the fact that the resonant peaks coalesce to the same frequency in the spectrum. Moreover, the frequency locking is also accompanied by a notable reduction of the full width at half maximum (FWHM) which reduces from $20 \mathrm{~Hz}$ (oscillator 1) and $32 \mathrm{~Hz}$ (oscillator 2) to $10 \mathrm{~Hz}$ in the frequency locked system. This indicates the improved immunity of the synchronized system to internal phase noise, and agrees with the phase noise reduction shown in several other coupled and self-injection locked oscillators systems ${ }^{38-41}$.

Figure 2a shows the evolution of the synchronization state (coupled vs. uncoupled) of the two oscillators as a function of the injected white noise amplitude for two different values of supply voltage $\left(\mathrm{V}_{\mathrm{DD}}=3 \mathrm{~V}\right.$ and $\left.4 \mathrm{~V}\right)$. It can be observed that in both the cases a minimum threshold noise voltage $\left(\mathrm{V}_{\mathrm{T}, \mathrm{NOISE}}\right)$ is required to induce frequency locking. Moreover, this critical noise voltage increases with the amplitude of the oscillator output (achieved by increasing $\mathrm{V}_{\mathrm{DD}}$ ). This is further elaborated in Fig. $2 \mathrm{~b}$ which describes the minimum $\mathrm{V}_{\mathrm{T}, \mathrm{NOISE}}$ required as a function of the oscillator signal amplitude. $\mathrm{V}_{\mathrm{T}, \mathrm{NOISE}}$ increases linearly with the oscillator amplitude indicating that a minimum signal to (input) noise ratio $(\sim 9: 1)$ is required to induce frequency locking. Further, the relative phase difference $(\varnothing)$ between the oscillators, frequency locked by noise (Fig. 2c), evolves with the noise amplitude. Here, phase is defined using the relative time difference between the voltage troughs of the waveforms. Each oscillation is considered as a phase change of $2 \pi$ radians $\left(\equiv 360^{\circ}\right)$ and the relative phase difference is defined as: $\Delta \Phi=\left(\frac{\Delta t}{T}\right) \times 360^{\circ}(\Delta \mathrm{t}$ : minimum time difference between the adjacent troughs of the two oscillators; T: time period). Oscillator 2 (orange in Fig. 1b), with higher stand-alone resonant frequency, initially leads oscillator 1 (green) until they exhibit nearly anti-phase locking; beyond this point, oscillator 2 lags oscillator 1.

Synchronization of a larger oscillator system. Further, we also evaluate experimentally the synchronization of a larger system of up to 4 oscillators using white noise injection (Fig. 2d). It can be observed that the noise injection not only enables frequency locking among the oscillators but the critical noise voltage $\left(\mathrm{V}_{\mathrm{T}, \mathrm{NOISE}}\right)$ 
reduces with increasing number of oscillators. This can be attributed to the reduced internal phase noise which exhibits an inverse dependence on the number of oscillators in the system ${ }^{42,43}$.

Noise induced synchronization in coupled oscillators. While the uncoupled oscillator system (synchronized by noise) illustrates how noise promotes synchronization, its effect on the dynamics of a coupled system of oscillators is particularly relevant to computational applications. Consequently, we explore the effect of noise on the frequency locking dynamics of coupled oscillators and their resulting computational properties relevant to solving the graph coloring problem.

The graph coloring problem entails computing the minimum number of colors (labels) required to be assigned to the nodes of a graph such that no two nodes having a common edge are assigned the same color. The problem is NP-hard and is still considered intractable to solve using digital computers, motivating the exploration of alternate approaches. This problem can be elegantly mapped to the oscillator hardware by creating a topologically equivalent coupled oscillator network (graph node $\equiv$ oscillator, and edge $\equiv$ coupling capacitor $\mathrm{C}_{\mathrm{C}}$ ). Subsequently, when the coupling strength is strong enough to frequency lock the oscillators to a common frequency, the resulting phase dynamics exhibit a unique phase ordering such that clusters of nodes without an edge (independent set) and can be assigned the same color, appear consecutively in the circular ordering. The nodes of the same color can then be separated using a simple polynomial time sorting algorithm. Details of this approach have been discussed and demonstrated in our prior work ${ }^{11}$. It is important to emphasize that a critical coupling strength indicated by the magnitude of the coupling capacitance is required to induce frequency locking among the oscillators and observe the desired phase dynamics.

To understand the effect of external noise injection, we first evaluate the minimum coupling strength required to induce synchronization. Figures $3 b, c$ shows the frequency spectrum of the oscillators for the illustrative graph in Fig. $3 \mathrm{a}$ for $\mathrm{C}_{\mathrm{C}}=1 \mathrm{pF}$ and $5 \mathrm{pF}$, respectively. In the absence of external noise, a minimum $\mathrm{C}_{\mathrm{C}}=5 \mathrm{pF}$ is required to induce synchronization as shown in Fig. 3c. The corresponding time domain waveforms of the frequency locked oscillators and the phase plots, shown in Fig. 3 e, demonstrate a cyclic phase ordering $(1,2,4,3,1 \ldots)$ where independent nodes (i.e. without an edge; 2,4 here) appear consecutively. Using simple post processing, the nodes can be separated into different clusters $(=3$, in this problem) of independent sets $(\{1\},\{2,4\},\{3\})$ each of which can be assigned an independent color. Thus, the solution to the graph in Fig. 3a is equal to 3 (colors).

However, the oscillators fail to exhibit frequency locking when $\mathrm{C}_{C}<5 \mathrm{pF}$. The minimum coupling strength requirement puts a constraint on the minimum size and area of the coupling element. Since the number of elements in the coupling network of a reconfigurable coupled oscillator based computational platform exhibits a square law dependence $(=P(\mathrm{~N}, 2))$ on the number of oscillators $(\mathrm{N})$, a large footprint for an individual element can dramatically limit the platform's scalability and the reconfigurability.

However, when white noise of appropriate amplitude $\left(180 \mathrm{mV}_{\mathrm{RMS}}\right)$ is injected (Fig. $\left.3 \mathrm{~d}\right)$, it can be observed that the coupled oscillators not only exhibit frequency locking but also show the optimal phase ordering $(\{1\},\{3\}$, $\{2,4\})$, giving rise to the optimal coloring solution at a significantly lower $C_{C}(=1 p F)(F i g .3 d, f)$. It is to be noted that even though the relative ordering observed in Fig. $3 \mathrm{f}$ is different from Fig. 3e, the ordering is still optimal.

We also experimentally evaluate various coupled oscillator network configurations shown in Fig. 4 . In all the configurations, a minimum threshold of $\mathrm{C}_{C}=5 \mathrm{pF}$ is required to induce frequency locking without the external injection of noise. However, the injection of noise helps induce frequency locking at a lower value of $\mathrm{C}_{\mathrm{C}}=1 \mathrm{pF}$, thus, facilitating a $5 \times$ reduction in the minimum required coupling capacitance. Subsequently, this property can be leveraged to proportionally reduce the area required for implementing the coupling capacitor and thus, help the scaling of the area-intensive coupling architecture.

Finally, we evaluate using LT-SPICE simulations, the ability to extend this approach to solving larger graphs. The inset in Fig. 5 shows a schematic of the $k=4$ nearest neighbor graphs ${ }^{44}$ of various sizes up to 64 nodes evaluated here. The same Schmitt trigger oscillator design was considered, and the oscillator dynamics were simulated over a time period of $10 \mathrm{~ms}$ wherein the system was observed to always attain steady state; multiple runs $(>10)$ were performed for each graph. Subsequently, the relative phase difference among the oscillators is used to construct the phase ordering and compute the coloring solution. Figure 5 compares the simulated graph coloring solution obtained using the oscillators with larger coupling capacitance $\left(C_{C}=50 \mathrm{pF}\right)$ and without injected noise, with that obtained using smaller coupling capacitance and external noise injection (minimum $\mathrm{V}_{\text {NOISE }}$ required is between $60 \mathrm{mV}_{\mathrm{RMS}}$ (64 oscillators) to $120 \mathrm{mV}_{\mathrm{RMS}}$ (8 oscillators)); the optimal solution is also shown for reference. It can be observed that the oscillators with externally injected noise (and lower $\mathrm{C}_{\mathrm{C}}$ ) not only exhibit frequency locking (no frequency locking is observed in the absence of noise when $\mathrm{C}_{\mathrm{C}}=0.5 \mathrm{pF}$ ) but also produce the same (optimal) solution as those without external noise injection (but higher $\mathrm{C}_{\mathrm{C}}$ ). This further supports the experimental observation that the injection of external noise into the coupled system lowers the minimum coupling capacitance threshold while facilitating the phase dynamics relevant to computation.

\section{Discussion}

In summary, we elucidate the critical role of injected noise in the synchronization dynamics of uncoupled and coupled (but not frequency locked) oscillators. Moreover, our work demonstrates empirically that noise reduces the minimum coupling strength required to realize the computational properties of the oscillators for solving combinatorial optimization problems such as graph coloring, thus, enabling an additional 'knob' to optimize the implementation and scalability of the area-hungry coupling network in an oscillator platform. Finally, these results also motivate the exploration of the role of noise on the computational performance of other non-Boolean dynamical systems such as spiking neural networks. 


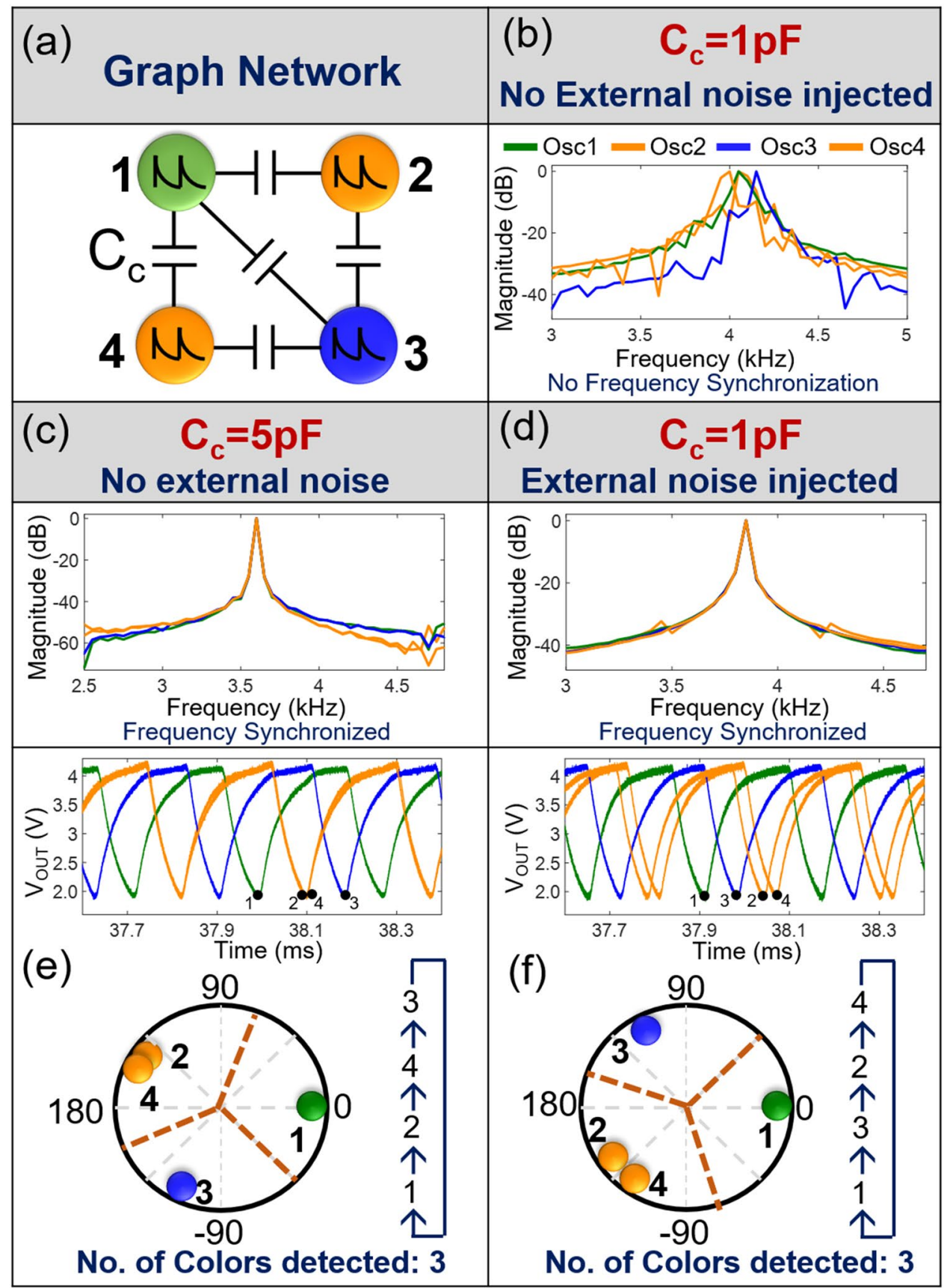

Figure 3. (a) Schematic of the oscillator network. Frequency spectrum of the four coupled oscillators with: (b) $\mathrm{C}_{\mathrm{c}}=1 \mathrm{pF}$; no frequency locking without injection of noise (c) $\mathrm{C}_{\mathrm{c}}=5 \mathrm{pF}$, where oscillators exhibit frequency locking owing to the larger value of coupling capacitance; $(\mathbf{d}) \mathrm{C}_{\mathrm{c}}=1 \mathrm{pF}$, where the noise induced frequency locking is observed. (e) and (f) Time domain waveforms corresponding to (c) and (d), respectively, along with the phase plots showing the correct phase ordering that gives rise to the optimal graph coloring solution. 


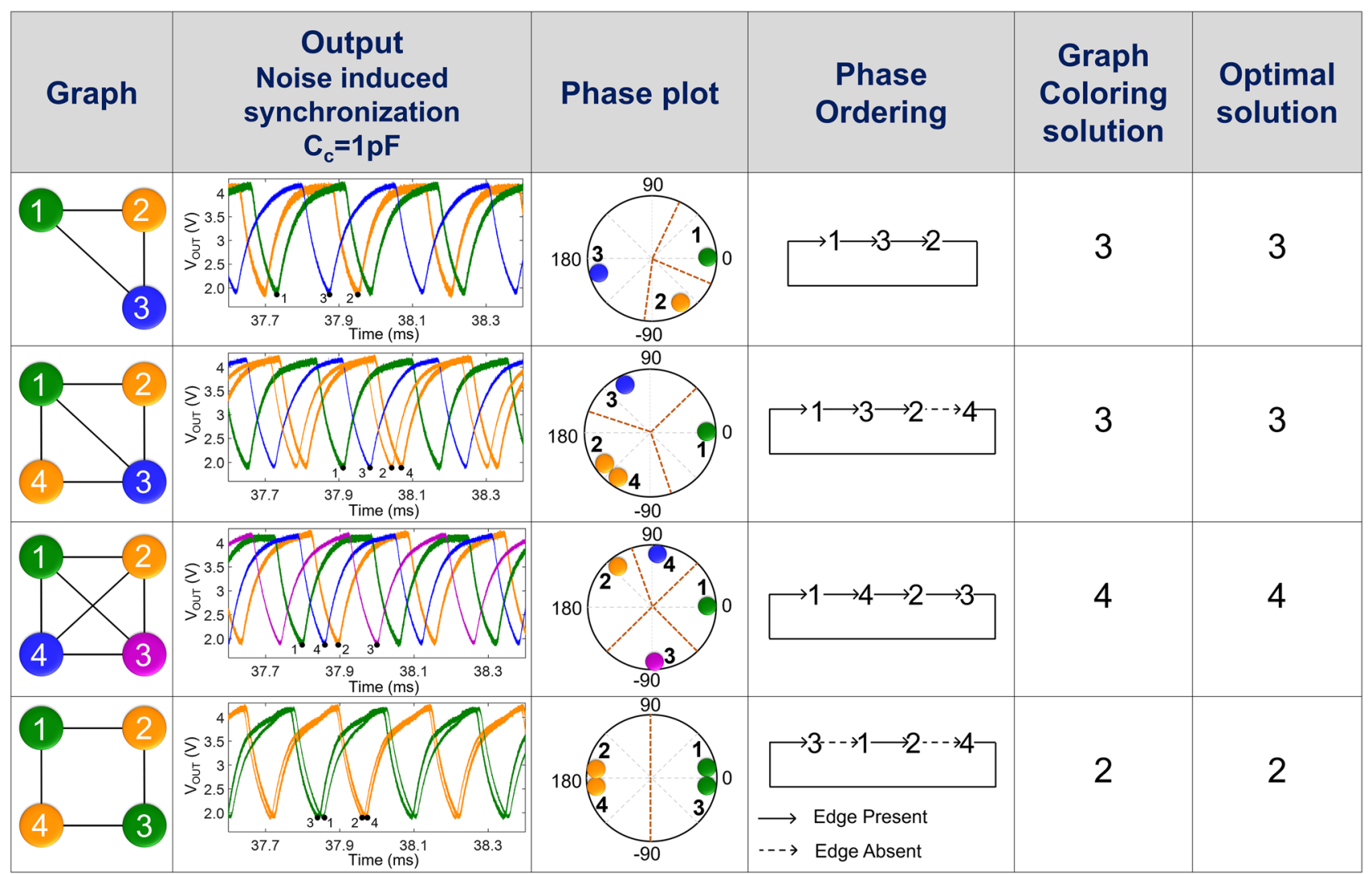

Figure 4. Experimentally observed noise induced synchronization, resulting phase plot and observed graph coloring solutions for various coupled oscillator networks $\left(\mathrm{C}_{\mathrm{C}}=1 \mathrm{pF}\right)$.

\begin{tabular}{|c|c|c|c|}
\hline $\begin{array}{c}\mathrm{K}=4 \\
\text { Nearest } \\
\text { Neighbor } \\
\text { Graphs } \\
\mathrm{N}\end{array}$ & $\begin{array}{c}\text { Best Solution } \\
-\mathrm{C}_{\mathrm{c}}=50 \mathrm{pF} \\
- \text { No noise }\end{array}$ & $\begin{array}{c}\text { Best Solution } \\
-\mathrm{C}_{\mathrm{c}}=0.5 \mathrm{pF} \\
\text {-With noise injection }\end{array}$ & $\begin{array}{c}\text { Optimal Solution } \\
(=\mathrm{N} \% 3+3)\end{array}$ \\
\hline 8 & 5 & 5 & 5 \\
\hline 32 & 4 & 4 & 5 \\
\hline 64 & 5 & 5 & 4 \\
\hline
\end{tabular}

Figure 5. Simulations showing coloring of $K=4$ nearest neighbor graphs of various size $(\mathrm{N})$ using the coupled oscillators, with and without the injection of noise $\left(180 \mathrm{mV}_{\mathrm{RMS}}\right)$. It can be observed that the injection of noise reduces the required capacitance to observe frequency locking and the corresponding computation relevant phase properties. $C_{C}=50 \mathrm{pF}$ (without noise) and $\mathrm{C}_{C}=0.5 \mathrm{pF}$ (with noise) were chosen since stable frequency locking at these values for all the analyzed graphs. With $\mathrm{C}_{\mathrm{C}}=0.5 \mathrm{pF}$, the system does not exhibit synchronization before the injection of noise. 
Received: 28 October 2020; Accepted: 8 February 2021

Published online: 24 February 2021

\section{References}

1. Csaba, G. \& Porod, W. Coupled oscillators for computing: a review and perspective. Appl. Phys. Rev. 7, 011302 (2020).

2. Csaba, G., Raychowdhury, A., Datta, S. \& Porod, W. Computing with coupled oscillators: Theory, devices, and applications. In 2018 IEEE International Symposium on Circuits and Systems (ISCAS) 1-5. https://doi.org/10.1109/ISCAS.2018.8351664 (2018).

3. Nikonov, D. E. et al. Coupled-oscillator associative memory array operation for pattern recognition. IEEE J. Explor. Solid-State Comput. Devices Circuits 1, 85-93 (2015).

4. Lee, D. et al. $\mathrm{NbO}_{2}$-based frequency storable coupled oscillators for associative memory application. IEEE J. Electron Devices Soc. 6, 250-253 (2018).

5. Sharma, A. A., Kesim Y., Shulaker, M., Kuo, C., Augustine C., Wong, H. S. -P., Mitra, S., Skowronski. M., Bain, J. A. \& Weldon, J. A. Low-power, high-performance S-NDR oscillators for stereo (3D) vision using directly-coupled oscillator networks. In 2016 IEEE Symposium on VLSI Technology, 1-2 (IEEE, 2016). https://doi.org/10.1109/VLSIT.2016.7573438.

6. Nikonov, D. E., Kurahashi, P., Ayers, J. S., Lee, H.-J., Fan, Y. \& Young, I. A. A Coupled CMOS Oscillator Array for 8ns and 55pJ Inference in Convolutional Neural Networks. Preprint at http://arXiv.org/1910.11803 (2019).

7. Cotter, M., Fang, Y., Levitan, S., Chiarulli, D. \& Narayanan, V. Computational Architectures Based on Coupled Oscillators. In 2014 IEEE Computer Society Annual Symposium on VLSI (ISVLSI), 130-135 (2014). https://doi.org/10.1109/ISVLSI.2014.87.

8. C Corti, E., Gotsmann, B., Moselund, K., Stolichnov, Igor., Ionescu, A., Karg, Siegfried. Resistive Coupled $\mathrm{VO}_{2}$ Oscillators for Image Recognition. In 2018 IEEE International Conference on Rebooting Computing (ICRC). 1-7 (IEEE, 2018). https://doi.org/10.1109/ ICRC.2018.8638626.

9. Csaba, G., Papp, A., Porod, W. \& Yeniceri, R. Non-boolean computing based on linear waves and oscillators. Eur. Solid-State Device Res. Conf. 2015, 101-104. https://doi.org/10.1109/ESSDERC.2015.7324723 (2015).

10. Cosp, J. \& Madrenas, J. Scene segmentation using neuromorphic oscillatory networks. IEEE Trans. Neural Networks 14, 1278-1296 (2003).

11. Parihar, A., Shukla, N., Jerry, M., Datta, S. \& Raychowdhury, A. Vertex coloring of graphs via phase dynamics of coupled oscillatory networks. Sci. Rep. 7, 1-11 (2017).

12. Mallick, A. et al. Using synchronized oscillators to compute the maximum independent set. Nat. Commun. 11, 4689 (2020).

13. Wang, T. \& Roychowdhury, J. OIM: oscillator-based Ising machines for solving combinatorial optimisation problems. Lect. Notes Comput. Sci. Subser. Lect. Notes Artif. Intell. Lect. Notes Bioinform. 11493 LNCS, 232-256 (2019).

14. Chou, J., Bramhavar, S., Ghosh, S. \& Herzog, W. Analog coupled oscillator based weighted Ising machine. Sci. Rep. 9, 1-10 (2019).

15. Dutta, S. et al. Experimental demonstration of phase transition nano-oscillator based Ising machine. Tech. Dig. Int. Electron Devices Meet. IEDM 911, 914. https://doi.org/10.1109/IEDM19573.2019.8993460 (2019).

16. Bashar, M.K., Mallick, A., Truesdell, D.S., Calhoun, B.H., Joshi, S. \& Shukla, N. Experimental Demonstration of a Reconfigurable Coupled Oscillator Platform to Solve the Max-Cut Problem. IEEE Journal on Exploratory Solid-State Computational Devices and Circuits 6, 116 (2020).

17. Ulmann, B. Analog Computing (Oldenbourg Wissenschaftsverlag, Munich, 2013).

18. Pikovskii, A. S. Synchronization and stochastization of array of self-excited oscillators by external noise. Radiophys. Quantum Electron. 27, 390-395 (1984).

19. Goldobin, D. S. \& Pikovsky, A. S. Synchronization of self-sustained oscillators by common white noise. Phys. A 351, 126-132 (2005).

20. Kawamura, Y. Collective phase dynamics of globally coupled oscillators: noise-induced anti-phase synchronization. Phys. D Nonlinear Phenom. 270, 20-29 (2014).

21. Kurebayashi, W., Fujiwara, K. \& Ikeguchi, T. Colored noise induces synchronization of limit cycle oscillators. EPL 97, 50009 (2012).

22. Nagai, K. H. \& Kori, H. Noise-induced synchronization of a large population of globally coupled nonidentical oscillators. Phys. Rev. E Stat. Nonlinear Soft Matter Phys. 81, 065202 (2010).

23. Pimenova, A. V., Goldobin, D. S., Rosenblum, M. \& Pikovsky, A. Interplay of coupling and common noise at the transition to synchrony in oscillator populations. Sci. Rep. 6, 38518 (2016).

24. Nakada, K., Yakata, S. \& Kimura, T. Noise-induced synchronization in spin torque nano oscillators. J. Appl. Phys. 111, 07C920 (2012).

25. Kawamura, Y. \& Nakao, H. Optimization of noise-induced synchronization of oscillator networks. Phys. Rev. E 94, 032201 (2016).

26. Kawai, K. et al. Common noise-induced synchronization of relaxation oscillators. IFAC-PapersOnLine 48, 233-238 (2015).

27. Lai, Y. M. \& Porter, M. A. Noise-induced synchronization, desynchronization, and clustering in globally coupled nonidentical oscillators. Phys. Rev. E 88, 012905 (2013).

28. Nagai, K. \& Nakao, H. Experimental synchronization of circuit oscillations induced by common telegraph noise. Phys. Rev. E 79, 036205 (2009).

29. Ishimura, K. \& Tokuda, I. T. Limited Effect of Noise Injection on Synchronization of Crystal Oscillators. J. Circuits, Syst. Comput. 29, $2050026(2020)$.

30. Wang, W., Kiss, I. Z. \& Hudson, J. L. Experiments on arrays of globally coupled chaotic electrochemical oscillators: synchronization and clustering. Chaos An Interdiscip. J. Nonlinear Sci. 10, 248-256 (2000).

31. Du-Qu, W. \& Xiao-Shu, L. Coherence resonance and noise-induced synchronization in Hindmarsh-Rose neural network with different topologies. Commun. Theor. Phys. 48(4), 759 (2007).

32. Wang, Y., Chik, D. T. \& Wang, Z. D. Coherence resonance and noise-induced synchronization in globally coupled Hodgkin-Huxley neurons. Phys. Rev. E 61(1), 740 (2000).

33. Hauschildt, B., Janson, N. B., Balanov, A. \& Schöll, E. Noise-induced cooperative dynamics and its control in coupled neuron models. Phys. Rev. E 74(5), 051906 (2006).

34. Mainen, Z. \& Sejnowski, T. Reliability of spike timing in neocortical neurons. Science (80) 268, 1503-1506 (1995).

35. Nakamura, O. \& Tateno, K. Random pulse induced synchronization and resonance in uncoupled non-identical neuron models. Cogn. Neurodyn. 13, 303-312 (2019).

36. Banerjee, S. \& Ariffin, M. R. K. Noise induced synchronization of time-delayed semiconductor lasers and authentication based asymmetric encryption. Opt. Laser Technol. 45, 435-442 (2013).

37. Yamamoto, T. et al. Common-chaotic-signal induced synchronization in semiconductor lasers. Opt. Express 15, 3974 (2007).

38. Rohde, U.L., Poddar, A.K., Schoepf, J., Rebel, R. \& Patel, P. Low noise low cost ultra wideband N-push VCO. In IEEE MTT-S International Microwave Symposium Digest, 1171-1174. (IEEE, 2005). https://doi.org/10.1109/MWSYM.2005.1516884.

39. Rohde, U. L., Rebel, R. \& Poddar, A. K., Synergy Microwave Corp. Integrated low noise microwave wideband push-push VCO. U.S. Patent 7,088,189 (2006).

40. Poddar, A. K., Rohde, U. L. \& Daryoush, A. S., Synergy Microwave Corp. Integrated production of self injection locked self phase loop locked optoelectronic oscillator. U.S. Patent 9,094,133 (2015).

41. Poddar, A. K., Rohde, U. L. \& Daryoush, A. S., Synergy Microwave Corp. Self injection locked phase locked looped optoelectronic oscillator. U.S. Patent 9,088,369 (2015). 
42. Pikovsky, A., Rosenblum, M., Kurths, J. \& Hilborn, R. C. Synchronization: a universal concept in nonlinear science. Am. J. Phys. 70, 655-655 (2002).

43. Chang, H.-C., Cao, X., Vaughan, M. J., Mishra, U. K. \& York, R. A. Phase noise in externally injection-locked oscillator arrays. IEEE Trans. Microw. Theory Tech. 45, 2035-2042 (1997).

44. Weisstein, E. W. Möbius Ladder. Available at: https://mathworld.wolfram.com/MoebiusLadder.html.

Acknowledgements

This research was supported in part by the National Science Foundation (Grant No. 1914730).

\section{Author contributions}

J.V. performed the experiments, simulations and analyzed the data. M.K.B. helped with the performance of the experiments. N.S. supervised the study. J.V., N.S. wrote the manuscript. All authors discussed the results and commented on the manuscript.

\section{Competing interests}

The authors declare no competing interests.

\section{Additional information}

Correspondence and requests for materials should be addressed to N.S.

Reprints and permissions information is available at www.nature.com/reprints.

Publisher's note Springer Nature remains neutral with regard to jurisdictional claims in published maps and institutional affiliations.

(c) (i) Open Access This article is licensed under a Creative Commons Attribution 4.0 International

License, which permits use, sharing, adaptation, distribution and reproduction in any medium or format, as long as you give appropriate credit to the original author(s) and the source, provide a link to the Creative Commons licence, and indicate if changes were made. The images or other third party material in this article are included in the article's Creative Commons licence, unless indicated otherwise in a credit line to the material. If material is not included in the article's Creative Commons licence and your intended use is not permitted by statutory regulation or exceeds the permitted use, you will need to obtain permission directly from the copyright holder. To view a copy of this licence, visit http://creativecommons.org/licenses/by/4.0/.

(C) The Author(s) 2021 